\title{
Paolo Tortonese, Drogue, morale et morphologie: questions autour de Gautier et de Baudelaire
}

\section{Annalisa Bottacin}

\section{(2) OpenEdition}

1 Journals

\section{Edizione digitale}

URL: http://journals.openedition.org/studifrancesi/7653

DOI: 10.4000/studifrancesi.7653

ISSN: 2421-5856

\section{Editore}

Rosenberg \& Sellier

\section{Edizione cartacea}

Data di pubblicazione: 1 décembre 2009

Paginazione: 653

ISSN: 0039-2944

\section{Notizia bibliografica digitale}

Annalisa Bottacin, «Paolo Tortonese, Drogue, morale et morphologie: questions autour de Gautier et de Baudelaire», Studi Francesi [Online], 159 (LIII | III) | 2009, online dal 30 novembre 2015, consultato il 09 janvier 2021. URL: http://journals.openedition.org/studifrancesi/7653 ; DOI: https://doi.org/10.4000/ studifrancesi.7653

Questo documento è stato generato automaticamente il 9 janvier 2021.

\section{(c) (1)}

Studi Francesi è distribuita con Licenza Creative Commons Attribuzione - Non commerciale - Non opere derivate 4.0 Internazionale. 


\title{
Paolo Tortonese, Drogue, morale et morphologie: questions autour de Gautier et de Baudelaire
}

\author{
Annalisa Bottacin
}

\section{NOTIZIA}

PAOLO TORTONESE, Drogue, morale et morphologie: questions autour de Gautier et de Baudelaire, in Image et pathologie au XIX ${ }^{e}$ siècle, sous la direction de Paolo TORTONESE, «Cahiers de Littérature française» VI, Bergamo, University Press - Paris, L'Harmattan, 2008, pp. 133-154.

1 La questione posta da Paolo Tortonese nell'elaborazione di questo interessante saggio converge sul fatto se l'uso di droghe nel percorso artistico miri ad alimentare la creatività o piuttosto sia da considerarsi quale espressione di una moda, che pur incuriosendo, non viene seguita. Par questo essere il caso di Théophile Gautier, che ben si dilunga in tema nel descrivere le sue serate all'hôtel Pimodan; lo stesso Claude Pichois si dimostra molto dubbioso sull'effettiva assunzione di sostanze stupefacenti da parte di questo «bourgeois de province», che non è certo da vedersi come un consumatore di hashish. Da tale presupposto e nel confronto con Baudelaire, Tortonese avvia la sua dettagliata analisi e per meglio evidenziare quanto affermato si propone un'indagine esaminando in parallelo i lavori di Gautier, Le Hachisch (1843), Le Club des hachischins (1846) e quelli di Charles Baudelaire, Du Vin et du hachisch (1851) e Le Poème $d u$ hachisch (1858), sottolineandone in particolar modo le analogie strutturali e descrivendo la sintomatologia dell'ivresse. Il tutto accompagnato da una campionatura di testi dei due scrittori, il che ben evidenzia le differenziazioni e le simmetrie nella scoperta e nel susseguente percorso verso i paradis artificiels. E soffermandosi altresì sulle tesi di Jacques Moreau de Tours, che in Du Hachich et de l'aliénation mentale del 1845, aveva sostenuto che «le hachich laisse à celui qui se soumet à son étrange influence le 
pouvoir d'étudier sur lui-même les désordres moraux qui caractérisent la folie» (p. 137).

Dopo un discorso generale, nella seconda parte del saggio, Tortonese rileva come i punti di contatto tra Gautier et Baudelaire siano comunque numerosi, in particolar modo «dans la manière de saisir les modalités du délire» e li classifica in tre categorie: «la déformation, la transposition, la fusion» (p.137), appoggiandosi a varie citazioni tratte dai lavori succitati dei due scrittori. Lo studioso evidenzia inoltre che si tratta di testi d'indubbia importanza sulle reazioni all'hashish, in quanto hanno non pochi punti in comune con le teorie scientifiche del dottor Alexandre Brierre de Boismont, autore di un articolo, pubblicato il 2 maggio 1840, sulla "Gazette médicale de Paris", che relaziona di una seduta sperimentale di hashish che si sarebbe tenuta presso "A. de G.» (Ajasson de Grandsagne), allievo di Cuvier, che fu legato a George Sand. Vengono ripresi alcuni dei sintomi descritti sia da Gautier che da Baudelaire, come l'ilarità, l'estasi del kief, l'iperestesia, seppur non si parli di sinestesia. Moreau de Tours ha classificato le reazioni all'hashish in otto "fenomeni", che vengono qui rigorosamente presentati dal critico e associati alle visioni di Gautier e di Baudelaire, con molti interessanti esempi. In fase conclusiva, Tortonese studia quale sia la separazione netta, relativamente al presunto o vero uso di oppiacei, tra i due scrittori, cioè «la problématique morale» utilizzata "dans le sens du bien ou du mal, du péché, du châtiment, problématiques étrangères à Gautier et familières à Baudelaire» (p.147). Per concludere, par interessante citare questo giudizio di Gautier sull'autore delle Fleurs du mal, che ben supera il fenomeno dell'ivresse, in quanto l'immagine non è solo il frutto di corrispondenze segrete, bensì l'effetto prodotto dall'artifex stesso, ben all'interno di una propria luminosità, che produce il genio: «ll possède aussi le don de correspondance, pour employer le même idiome mystique, c'est-à-dire qu'il sait découvrir par une intuition secrète des rapports invisibles à d'autrui et rapprocher ainsi, par des analogies inattendues que seul le voyant peut saisir, les objets les plus éloignés et les plus opposés en apparence» (GAUTIER, «Charles Baudelaire», in Portraits littéraires, Paris, Aubry, 1943, p. 208). 